\title{
SOLUTION PHASE SYNTHESIS OF IMIDAZOLE- AND PYRROLE-CONTAINING HAIRPIN POLYAMIDES
}

\author{
Dorothy Harris, ${ }^{a}$ Michelle Stewart, ${ }^{a}$ Alan Sielaff, ${ }^{b}$ Keith Mulder, ${ }^{b}$ \\ Toni Brown, ${ }^{\mathrm{b}}$ Hilary Mackay, ${ }^{\mathrm{b}}$ Moses Lee ${ }^{\mathrm{a}, \mathrm{b}, *}$ \\ lee@hope.edu \\ ${ }^{a}$ Department of Chemistry, Furman University, Greenville, SC, 29613 \\ ${ }^{\mathrm{r}}$ Department of Chemistry, Hope College, Holland, MI, 49423
}

\begin{abstract}
Hairpin imidazole (I)- and pyrrole (P)-containing polyamide analogs of distamycin that bind at specific sequences in the minor groove of DNA are potentially useful gene control agents. There is a pressing need for efficient syntheses of such polyamides. The syntheses of four specific hexaheterocyclic hairpin polyamides (PIP- $\gamma$-PPP 1, PIP- $\gamma$-PII 2, PIP- $\gamma$-III 3, and PIP- $\gamma$-IPI 4, $\gamma$ represents 4-aminobutyrate) using a solution phase approach are reported.
\end{abstract}

\section{Introduction}

Heterocyclic polyamide compounds that bind to the minor groove of DNA in a sequence specific fashion are useful for regulating the expression of specific genes in cells. These compounds are potentially useful as DNA-targeted pharmaceutical agents. ${ }^{1}$ Distamycin A (Figure 1) is a polyamide that contains $\mathrm{N}$-methylpyrrole-2-carboxamido moieties and an amidine group at the $\mathrm{C}$-terminus. It binds in a stacked anti-parallel 2:1 (ligand:DNA) fashion to A.T rich sequences of DNA., Substitution of the pyrrole moieties with imidazoles within the polyamides has led to the development of a set of pairing rules for base pair recognition. ${ }^{1,4-6} \mathrm{~A}$ stacked $\mathrm{P} / \mathrm{P}$ pairing recognizes $A \cdot T$ or $T \cdot A, I / P$ binds to $G \cdot C, P / I$ targets $C \cdot G$, and $I / I$ binds selectively to $G \cdot T$ mismatches. ${ }^{5-7}$

Hairpin polyamides were designed to circumvent the need of simple polyamides to "find each other" within the nucleus of cells and bind in the minor groove as stacked dimers. ${ }^{1,8-10}$ Polyamides with two heterocyclic components linked together by a 4-aminobutyrate $(\gamma)$ moiety are prone to adopt a hairpin shape. In this conformation, the heterocyclyes are stacked forming the appropriate pyrrole and imidazole pairings for base pair recognition. ${ }^{1,8}$ Dervan $^{1,8}$ and others ${ }^{9,10}$ have demonstrated that hairpin polyamides are capable of recognizing their respective cognate sequences with high affinity. These compounds are able to elicit specific biological responses in cells through modulating gene expression. ${ }^{1,9,10}$ For example, JH-37 (Figure 1) is a hairpin polyamide that binds to the ICB 2 (3'- 

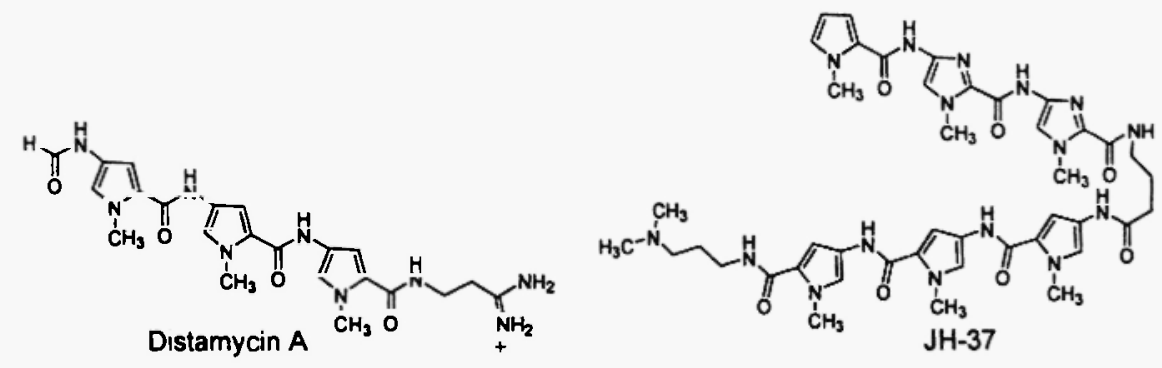

Figure 1. Structures of distamycin A and JH-37.

ATTGG-5') site of the topoisomerase II $\alpha$ promoter. ${ }^{10}$ This interaction results in up-regulation of the topoisomerase II $\alpha$ gene in confluent cancer cells. ${ }^{10}$ Hairpin polyamides are, therefore, interesting and important biologically active compounds. The syntheses of the hairpin polyamides reported by Dervan's group have been accomplished through a solid phase approach. ${ }^{11}$ However, our synthesis of JH-37 was accomplished through solution phase, which was flexible and required minimal equipment. We have further explored the solution phase synthesis of hairpin polyamides, and four new compounds (1-4) were designed and prepared. Each polyamide was designed to target specific DNA sequences based on the pairing rules. This communication is focused only on the synthesis of these compounds and on demonstrating the versatility of the solution phase approach. The biochemical properties of compounds 1-4 will be reported elsewhere.
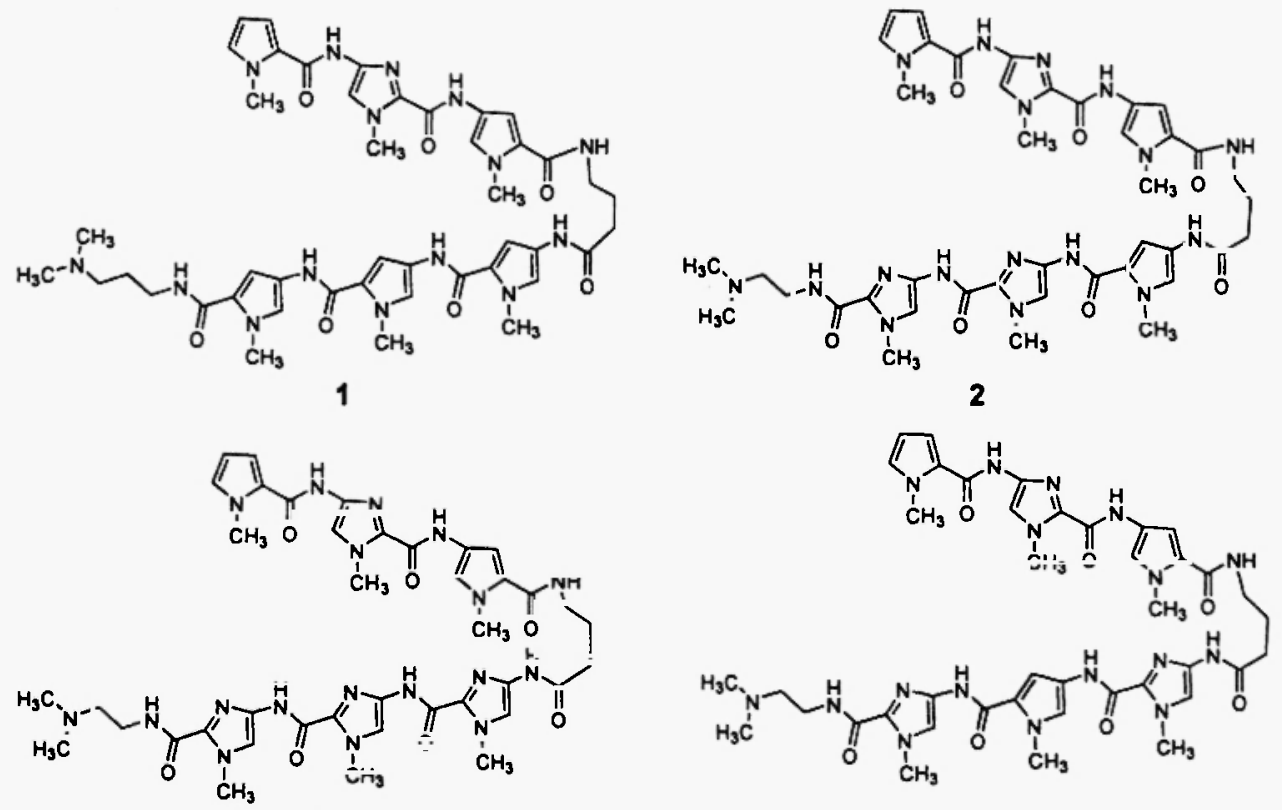

3

Figure 2. Structures of the four target hairpin polyamides 1-4. 


\section{Results and Discussion}

The synthesis of hairpin polyamides 1-4 (Figure 2) required compound 5, which was produced according to the strategy depicted in Scheme 1 . Reaction of trichlororacetylpyrrole $6,{ }^{12}$ with ethyl 4 aminobutylrate hydrochloride under refluxing conditions yielded the monoheterocyclic compound 7 in $60 \%$ yield. The nitro-group on compound 7 was reduced by catalytic hydrogenation over $5 \% \mathrm{Pd} / \mathrm{C}$ to give amine 8. Reaction of amine 8 imidazole acid chloride $9^{13,14}$ (formed by refluxing acid 10 with oxalyl chloride) gave the diheterocyclic compound 11 in good yield (82\%). Compound 11 was reduced as previously described and the amine intermediate was coupled to 1-methylpyrrole-2carboxylic acid in the presence of EDCI and DMAP to yield compound 12 in $61 \%$ yield. Compound 12 was hydrolyzed with aqueous $\mathrm{NaOH}(2 \mathrm{M})$ to yield compound 5 in $91 \%$ yield.

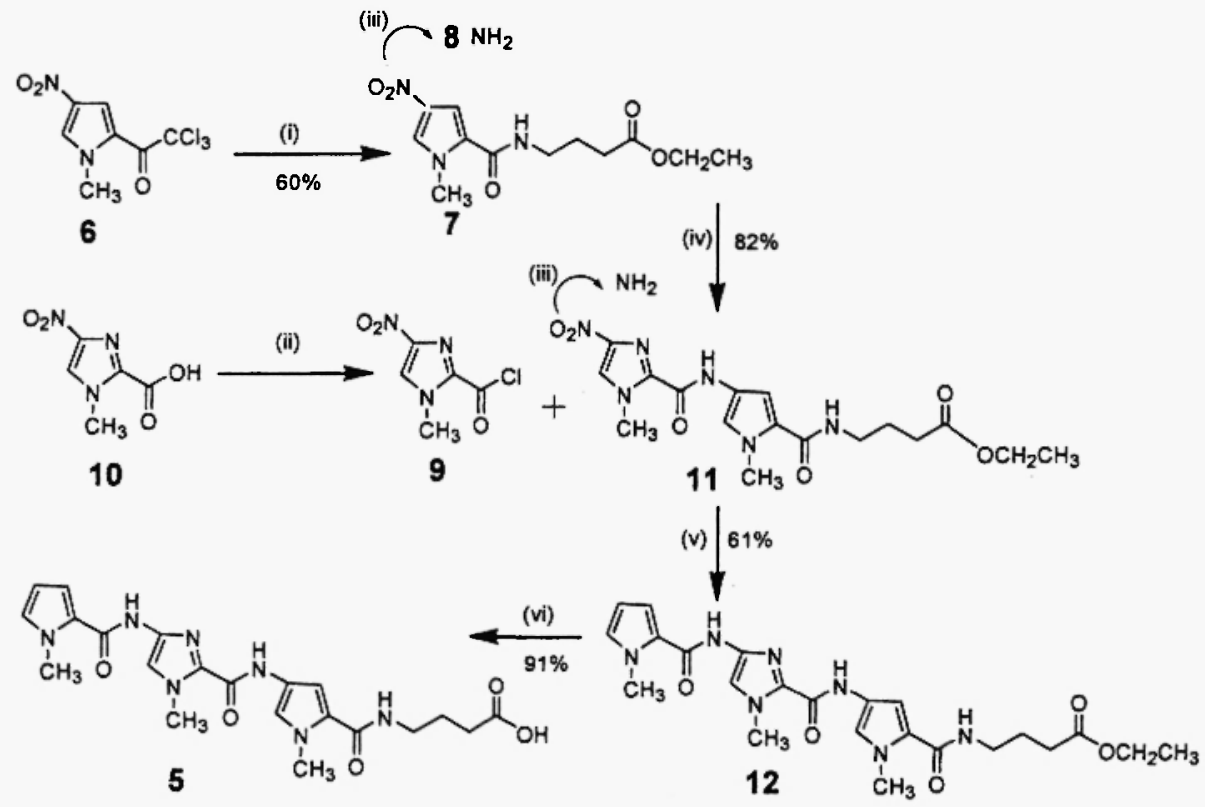

Scheme 1: i) ethyl 4-aminobutyrate hydrochloride, TEA, dry THF, reflux, $24 \mathrm{~h}$; ii) oxalyl chloride, dry THF, reflux, $50 \mathrm{~min}$; iii) $5 \% \mathrm{Pd} / \mathrm{C}$, cold EtOH, $\mathrm{H}_{2}, 24 \mathrm{~h}$; iv) 9, TEA, dry $\mathrm{CH}_{2} \mathrm{Cl}_{2}, 0^{\circ} \mathrm{C}-\mathrm{RT}, 24$ h; v) 1-methylpyrrole-2-carboxylic acid, DMAP, EDCI, dry DMF, RT, 24 h; vi) $\mathrm{THF}, \mathrm{H}_{2} \mathrm{O}, 2 \mathrm{M}$ aq. $\mathrm{NaOH}$, reflux, $6 \mathrm{~h}$.

The strategy of condensing compound 5 with the respective nitro-triheterocyclic compounds (1316) $)^{5-7,10,12,15}$ is shown in Scheme 2. Compounds 17 and 18 were formed in excellent yields (91 and $93 \%$, respectively) by reacting acid chlorides 9 and $19^{16}$ with N,N-dimethylethyleneamine and 3dimethylaminopropylamine. Acid chloride 19 was produced from reaction of acid 20 in refluxing 
thionyl chloride. ${ }^{13,16}$ Reduction of compounds 17 and 18 in $\mathrm{MeOH}$ over $5 \% \mathrm{Pd} / \mathrm{C}$ at atmospheric pressure gave the respective amines, which were coupled with the either acid chloride 9 or 19 to yield the diheterocylic compounds $\mathbf{2 1 - 2 3}$ in 69, 17 and 69\%, respectively. Compounds 21-23 were subsequently converted to the nitro-triheterocyclic compounds 13-16 in moderate to good yields (29, 36,59 and $65 \%$, respectively). Reduction of the nitro groups in compounds $13-16$, followed by coupling with carboxylic acid 5 using PyBOP, yielded the four hairpin compounds 1-4 $(5,34,30$, $22 \%$, respectively) after purification by silica gel column chromatography. The products were characterized by IR, $500 \mathrm{MHz}{ }^{\mathrm{I}} \mathrm{H}-\mathrm{NMR}$, mass spectrometry and accurate mass measurements.

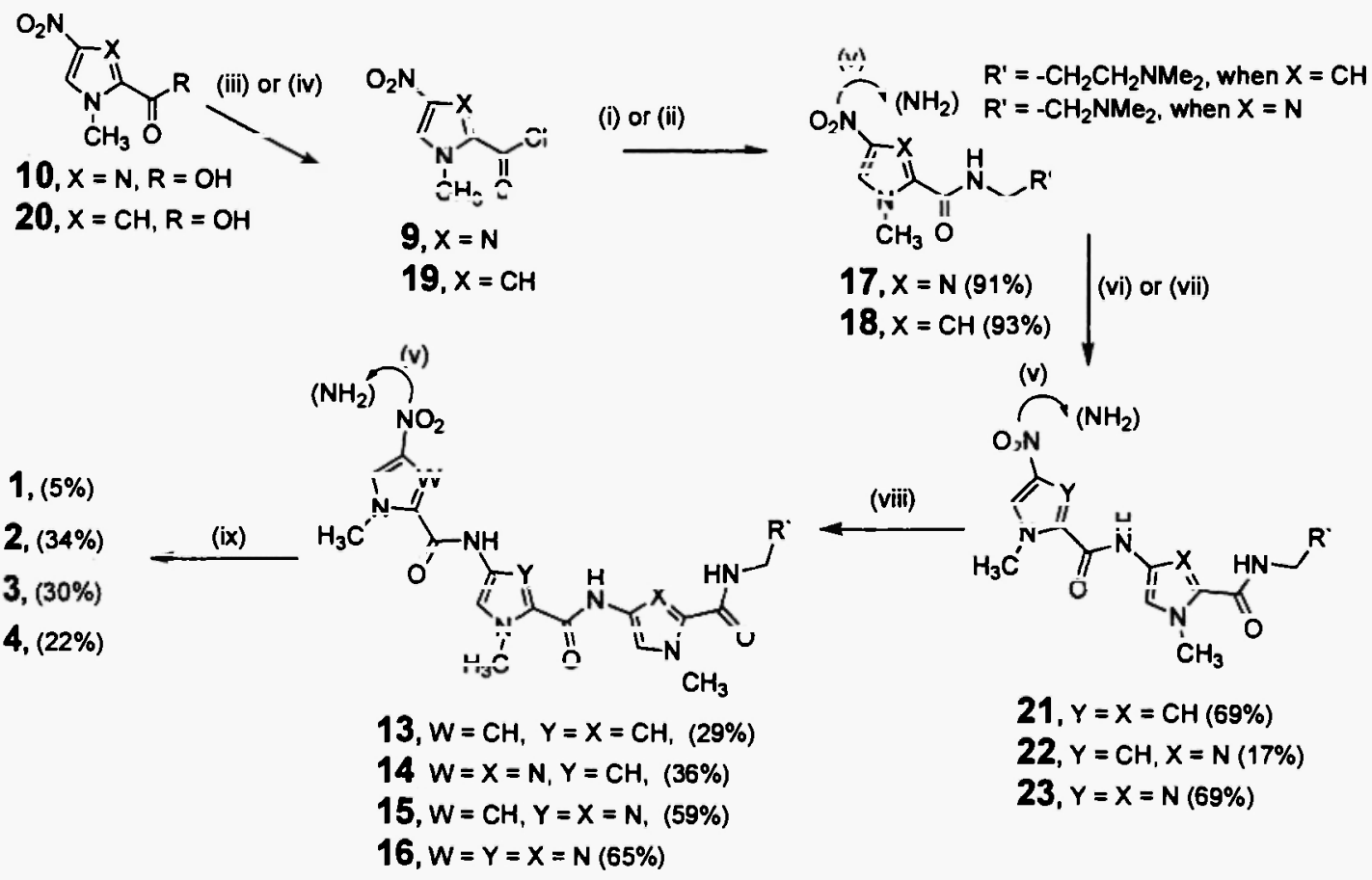

Scheme 2: i) 9 and 3-dimethylaminopropylamine, dry $\mathrm{CH}_{2} \mathrm{Cl}_{2}$, TEA, reflux, $24 \mathrm{~h}$; ii) $19 \mathrm{~N}, \mathrm{~N}$ dimethylethylenediamine, dry DCM, TEA, RT, 24 h iii) 10, oxalyl chloride, dry THF, reflux, 50 min; iv) 20, $\mathrm{SOCl}_{2}$, reflux, $15 \mathrm{~min}$; v) $5 \% \mathrm{Pd} / \mathrm{C}$, cold $\mathrm{MeOH}, \mathrm{H}_{2}, 24 \mathrm{~h}$; vi) 9, dry $\mathrm{CH}_{2} \mathrm{Cl}_{2}, \mathrm{TEA}, 0^{\circ} \mathrm{C}$

- RT, $24 \mathrm{~h}$; vii) 19, dry $\mathrm{CH}_{2} \mathrm{Cl}_{2}$, TEA, $0^{\circ} \mathrm{C}-\mathrm{RT}, 24 \mathrm{~h}$; viii) dry $\mathrm{CH}_{2} \mathrm{Cl}_{2}$, TEA, $0{ }^{\circ} \mathrm{C}-\mathrm{RT}, 24 \mathrm{~h}$; ix) 5, PyBOP, dry $\mathrm{CH}_{2} \mathrm{Cl}_{2}$, DIPEA, argon, $\mathrm{RT}, 192 \mathrm{~h}$.

\section{Experimental}

PIP- $\gamma$-COOH, Compound 5.

Compound 8. A solution of 4-nitro-2-trichlororacetylpyrrole $(6)^{11,12}(1.08 \mathrm{~g}, 3.68 \mathrm{mmol})$, ethyl 4aminobutylrate hydrochloride $(0.74 \mathrm{~g}, 4.42 \mathrm{mmol})$, triethylamine (TEA) $(1.13 \mathrm{~mL}, 8.10 \mathrm{mmol})$ in dry THF $(25 \mathrm{~mL})$ was heated to reflux for $24 \mathrm{~h}$. The solution was cooled to room temperature (RT) and concentrated. The brown residue was dissolved in $\mathrm{CHCl}_{3}$ and washed with $\mathrm{H}_{2} \mathrm{O}$ (subsequently basified to $\mathrm{pH} 10$ with aq. $\mathrm{NaOH}(5 \%))$. The aqueous phase was back-extracted using $\mathrm{CHCl}_{3}(2 \mathrm{x})$ and EtOAc (3x) and the organic layers were collected and combined, dried $\left(\mathrm{Na}_{2} \mathrm{SO}_{4}\right)$ and evaporated 
to dryness. Purification of the resulting residue was performed using flash-column chromatography $\left(100-90: 0-10 \% \mathrm{v} / \mathrm{v} \mathrm{CHCl}_{3} / \mathrm{MeOH}\right)$ to yield the ester (7) as a white/peach solid $(0.98 \mathrm{~g}, 60 \%)$, which was used directly. Amine 8 was produced from catalytic hydrogenation of ester 7 (4.67 mmol) over $5 \% \mathrm{Pd} / \mathrm{C}(100 \mathrm{mg})$ suspended in $\mathrm{MeOH}(50 \mathrm{~mL})$ at atmospheric pressure, room temperature and overnight. The catalyst was removed by vacuum filtration and the filtrate was concentrated, and the residue was coevaporated with dry $\mathrm{CH}_{2} \mathrm{Cl}_{2}$ twice and used immediately.

Compound 11. Acid chloride, $9^{13}$ (4.67 mmol), was dissolved in dry $\mathrm{CH}_{2} \mathrm{Cl}_{2}(15 \mathrm{~mL})$ and added dropwise to a solution of the amine $8(4.67 \mathrm{mmol})$, dry THF $(25 \mathrm{~mL})$, and TEA $(0.72 \mathrm{~mL}, 5.14$ $\mathrm{mmol}$ ) at $0{ }^{\circ} \mathrm{C}$. The reaction was stirred for $24 \mathrm{~h}$, evaporated to dryness and the residue dissolved in $\mathrm{CHCl}_{3}$, and washed with $\mathrm{H}_{2} \mathrm{O}$, The organic layer was collected, dried $\left(\mathrm{Na}_{2} \mathrm{SO}_{4}\right)$ and evaporated to dryness. Column chromatography $\left(100: 0 \% \mathrm{v} / \mathrm{v}, \mathrm{CHCI}_{3}\right)$ yielded compound 11 as a brown oil $(1.55 \mathrm{~g}$, $82 \%$ ): IR (neat) $3388,3113,2921,2847,1643,1530,1435,1384,1308 \mathrm{~cm}^{-1} ;{ }^{1} \mathrm{H}-\mathrm{NMR}\left(\mathrm{CDCI}_{3}, 500\right.$ $\mathrm{MHz}), 8.96(\mathrm{~s}, 1 \mathrm{H}), 7.98(\mathrm{t} \mathrm{br}, J=6.5 \mathrm{~Hz}, 1 \mathrm{H}), 7.83(\mathrm{~s}, 1 \mathrm{H}), 7.23(\mathrm{~d}, 1.5,1 \mathrm{H}), 6.45(\mathrm{~d}, J=1.5 \mathrm{~Hz}$, $1 \mathrm{H}), 4.20(\mathrm{~s}, 3 \mathrm{H}), 3.93(\mathrm{~s}, 3 \mathrm{H}), 3.46(\mathrm{q}, J=6.0 \mathrm{~Hz}, 2 \mathrm{H}), 2.46(\mathrm{t}, J=6.0 \mathrm{~Hz}, 2 \mathrm{H}, 2.31(\mathrm{~s}, 6 \mathrm{H}), 1.71$ (quintet, $J=6.0 \mathrm{~Hz}, 2 \mathrm{H}$ ).

Compound 12. Ester $11(250 \mathrm{mg}, 0.62 \mathrm{mmol})$ was reduced by catalytic hydrogenation over $5 \% \mathrm{Pd} / \mathrm{C}$ (100 mg) suspended in $\mathrm{MeOH}(100 \mathrm{~mL})$. Subsequent removal of the catalyst and concentration of the filtrate provided an amine intermediate, which was directly coupled to 1-methylpyrrole-2carboxylic acid (231 mg, $18.5 \mathrm{mmol}$ ) in the presence of DMAP (15 mg, $1.2 \mathrm{mmol})$ ), EDCI (354 mg, $18.5 \mathrm{mmol})$, and dissolved in dry DMF $(5 \mathrm{~mL})$. The reaction mixture was flushed with argon, protected from light, and stirred at $\mathrm{RT}$ for $24 \mathrm{~h}$. The DMF was removed via kügelrohr distillation (55 ${ }^{\circ} \mathrm{C}, 0.05 \mathrm{~mm} \mathrm{Hg}$ ). The residue was dissolved in $\mathrm{CHCl}_{3}$ and the same aqueous extraction procedure was performed as described for compound 7. Compound 12 was isolated following column chromatography $\left(90: 10 \% \mathrm{v} / \mathrm{v} \mathrm{CHCl}_{3} / \mathrm{MeOH}\right)$ as a yellow foam $(0.181 \mathrm{~g}, 61 \%)$ : IR (neat) 3392 , 3131, 2954, 2927, 2865, 1654, 1542, $1314 \mathrm{~cm}^{-1}$; ${ }^{1} \mathrm{H}-\mathrm{NMR}\left(\mathrm{CDCl}_{3}, 500 \mathrm{MHz}\right) 8.89$ (s, $\left.1 \mathrm{H}\right), 8.64$ (s, $1 \mathrm{H}), 7.98(\mathrm{~s}, 1 \mathrm{H}), 7.43(\mathrm{~s}, 1 \mathrm{H}), 7.27(\mathrm{~s}, 1 \mathrm{H}), 7.16(\mathrm{~d}, J-1.5 \mathrm{~Hz}, 1 \mathrm{H}), 6.79$ (dd, $J=4.5,2.0 \mathrm{~Hz}, 1 \mathrm{H})$, 6.57 (d, $J=2.0 \mathrm{~Hz}, 1 \mathrm{H}), 6.50$ (t, $J=4.5 \mathrm{~Hz}, 1 \mathrm{H}), 6.08$ (dd, $J=4.0,2.5 \mathrm{~Hz}, 1 \mathrm{H}), 3.99(\mathrm{~s}, 3 \mathrm{H}), 3.93$ $(\mathrm{s}, 3 \mathrm{H}), 3.85(\mathrm{~s}, 3 \mathrm{H}), 3.37$ (dd, $J-12.5,6.0 \mathrm{~Hz}, 2 \mathrm{H}), 2.37$ (t, $J=7.5 \mathrm{~Hz}, 2 \mathrm{H}), 1.87$ (quintet, $J=7.0$ $\mathrm{Hz}, 2 \mathrm{H}), 1.20$ (t, $J=7.5 \mathrm{~Hz}, 3 \mathrm{H}$ ); TOF-MS (electrospray) $\mathrm{m} / \mathrm{z}$ (relative intensity): $484\left(\mathrm{M}^{+} \mathrm{H}^{+}, 100\right)$.

Compound 5. Ester 12 (181 mg, $0.37 \mathrm{mmol})$ was dissolved in THF and water (1:1) (3 mL). Aq. $\mathrm{NaOH}(2 \mathrm{M}, 0.2 \mathrm{~mL}, 0.4 \mathrm{mmol})$ was added, and the solution was refluxed at $70-80{ }^{\circ} \mathrm{C}$ for $5 \mathrm{~h}$. Additional aq. $\mathrm{NaOH}(2 \mathrm{M}, 0.1 \mathrm{~mL}, 0.2 \mathrm{mmol})$ was added, and the solution was refluxed for one hour. The solvent was removed under reduced pressure. The residue was dissolved in water, cooled in an ice bath, and acidified with $3 \mathrm{M}$ aq. $\mathrm{HCl}(\mathrm{pH} 2)$. The cream powder was vacuum filtered and dried to give acid 5 (154 mg, 91\%), mp. $238^{\circ} \mathrm{C}$ : IR (Nujol) 3353, 3107, 1710, 1656, 1598, 1416, 1259, 1102, 802, $729 \mathrm{~cm}^{-1}$; ${ }^{1} \mathrm{H}-\mathrm{NMR}$ (DMSO-d $\left.6,500 \mathrm{MHz}\right) 12.01(\mathrm{~s}, 1 \mathrm{H}), 10.21(\mathrm{~s}, 1 \mathrm{H}), 9.86(\mathrm{~s}$, $1 \mathrm{H}), 8.03(\mathrm{~s}, 1 \mathrm{H}), 7.20(\mathrm{~s}, 1 \mathrm{H}), 7.10(\mathrm{~d}, J=4 \mathrm{~Hz}, 1 \mathrm{H}), 6.97(\mathrm{~s}, 1 \mathrm{H}), 6.95(\mathrm{~s}, 1 \mathrm{H}), 6.93(\mathrm{~s}, 1 \mathrm{H}), 6.05$ (dd, $J=0.75,2.5 \mathrm{~Hz}, 1 \mathrm{H}), 3.96(\mathrm{~s}, 3 \mathrm{H}), 3.88(\mathrm{~s}, 3 \mathrm{H}), 3.79(\mathrm{~s}, 3 \mathrm{H}), 3.18$ (dd, $J=7,11 \mathrm{~Hz}, 2 \mathrm{H}), 1.70$ $(\mathrm{t}, J=6.5 \mathrm{~Hz}, 2 \mathrm{H}) ;$ TOF-MS (electrospray, negative ion) $\mathrm{m} / \mathrm{z}$ (relative intensity): $454(\mathrm{M}-\mathrm{H}, 70)$.

\section{Hairpin PIP- $\gamma-P P P 1$.}

The nitro-tripyrrole $13^{12}(77.2 \mathrm{mg}, 0.16 \mathrm{mmol})$ was reduced by catalytic hydrogenation over $5 \%$ $\mathrm{Pd} / \mathrm{C}$ (50 mgs) suspended in $\mathrm{MeOH}(25 \mathrm{~mL})$ at atmospheric pressure, room temperature overnight. The catalyst was removed by suction filtration and the filtrate was concentrated. The residue was coevaporated with dry $\mathrm{CH}_{2} \mathrm{Cl}_{2}(2 \times 2 \mathrm{~mL})$ and the resulting amine was used directly in the next step. The amine, PyBOP (99.9 mg, $0.191 \mathrm{mmol})$, and acid 5 (91 $\mathrm{mg} ; 0.20 \mathrm{mmol}$ ) were suspended in dry 
$\mathrm{CH}_{2} \mathrm{Cl}_{2}(25 \mathrm{~mL})$ under an argon atmosphere. DIPEA $(0.07 \mathrm{~mL}, 0.4 \mathrm{mmol})$ was added to the suspension, and the reaction mixture stirred for 3 days under argon at $\mathrm{RT}$ and protected from light. The solvent was removed under reduced pressure and the residue dissolved in $\mathrm{CHCl}_{3} . \mathrm{H}_{2} \mathrm{O}$ [subsequently adjusted with aq. $\mathrm{NaOH}(2 \mathrm{M})(\mathrm{pH} 10)$ ] was added and the aqueous phase backextracted with $\mathrm{CHCl}_{3}(\mathrm{x} 2)$ and EtOAc (x2). The organic layers were combined, dried $\left(\mathrm{Na}_{2} \mathrm{SO}_{4}\right)$ and evaporated to dryness. Purification by column chromatography (gradient $\mathrm{CHCI}_{3}$, gradual increment of $\mathrm{MeOH}$ by $10 \%$ every $50 \mathrm{~mL}$ of solvent) yielded hairpin 1 as a brown solid $(7.4 \mathrm{mg}, 5.2 \%)$, mp. 271-275 ${ }^{\circ} \mathrm{C}$ : IR (Nujol): 3343, 3215, 1715, 1641, 1582, 1538, 1406, 1259, 1205, 1102, $734 \mathrm{~cm}^{-1}$; ${ }^{1} \mathrm{H}-$ NMR (500 MHz, DMSO-d $) 10.21(\mathrm{~s}, 1 \mathrm{H}), 9.89(\mathrm{~s}, 1 \mathrm{H}), 9.87(\mathrm{~s}, 1 \mathrm{H}), 9.83(\mathrm{~s}, 1 \mathrm{H}), 8.49(\mathrm{~s}, \mathrm{br}, 1 \mathrm{H})$, 8.06 (br t, 1H), 8.05 (br t, $1 \mathrm{H}), 7.54(\mathrm{~s}, 1 \mathrm{H}), 7.23(\mathrm{~s}, 1 \mathrm{H}), 7.22(\mathrm{~s}, 1 \mathrm{H}) 7.18(\mathrm{~s}, 1 \mathrm{H}), 7.10(\mathrm{~d}, J=8.5$ $\mathrm{Hz}, 1 \mathrm{H}), 7.03(\mathrm{~s}, 1 \mathrm{H}), 6.98(\mathrm{~s}, 1 \mathrm{H}), 6.90(\mathrm{~s}, 1 \mathrm{H}), 6.88(\mathrm{~s}, 1 \mathrm{H}), 6.82(\mathrm{~s}, 1 \mathrm{H}), 6.05(\mathrm{~d}, J=8.5 \mathrm{~Hz}, 1 \mathrm{H})$, $3.94(\mathrm{~s}, 3 \mathrm{H}), 3.88(\mathrm{~s}, 3 \mathrm{H}), 3.84(\mathrm{~s}, 3 \mathrm{H}), 3.83(\mathrm{~s}, 3 \mathrm{H}), 3.82(\mathrm{~s}, 3 \mathrm{H}), 3.79(\mathrm{~s}, 3 \mathrm{H}), 3.22(\mathrm{q}, J=6.5 \mathrm{~Hz}$, 2H) $3.18(\mathrm{q}, J=5.5 \mathrm{~Hz}, 2 \mathrm{H}), 2.28(\mathrm{t}, J=6.5 \mathrm{~Hz}, 2 \mathrm{H}), 2.24(\mathrm{t}, J=6.5 \mathrm{~Hz}, 2 \mathrm{H}), 1.80$ (quintet, $J=6.0$ $\mathrm{Hz}, 2 \mathrm{H}), 1.61$ (quintet, $J=6.0 \mathrm{~Hz}, 2 \mathrm{H}$ ); TOF-MS (electrospray) $\mathrm{m} / \mathrm{z}$ (relative intensity) $906\left(\mathrm{M}+\mathrm{H}^{+}\right.$, 30); Accurate mass for $\mathrm{C}_{44} \mathrm{H}_{56} \mathrm{~N}_{15} \mathrm{O}_{7}$ : calcd. 906.4487 , obsd. 906.4468 .

Hairpin PIP- $\gamma-P I I 2$.

Hairpin 2 was obtained as a yellow solid $(74.6 \mathrm{~g}, 34 \%), \mathrm{mp} .146-149^{\circ} \mathrm{C}$ : IR (neat) 33732925,2854 , 2688, 1649, 1534, 1457, 1410, $12491114,746 \mathrm{~cm}^{-1}$; ${ }^{1} \mathrm{H}-\mathrm{NMR}$ (DMSO-d ${ }^{6}, 500 \mathrm{MHz}$ ) 10.36 (br s, $1 \mathrm{H}), 10.20(\mathrm{~s}, 1 \mathrm{H}), 9.86(\mathrm{~s}, 1 \mathrm{H}), 9.84(\mathrm{~s}, 1 \mathrm{H}), 9.41(\mathrm{~s}, 1 \mathrm{H}), 9.41(\mathrm{br} \mathrm{s}, 1 \mathrm{H}), 8.21(\mathrm{br} \mathrm{s}, 1 \mathrm{H}), 8.05$ (br s, $1 \mathrm{H}), 7.61(\mathrm{~s}, 1 \mathrm{H}), 7.53(\mathrm{~s}, 1 \mathrm{H}), 7.27(\mathrm{~s}, 1 \mathrm{H}), 7.23(\mathrm{~s}, 1 \mathrm{H}), 7.09(\mathrm{~d}, J=2.5 \mathrm{~Hz}, 1 \mathrm{H}), 6.97(\mathrm{~s}, 1 \mathrm{H}), 6.95$ $(\mathrm{s}, 1 \mathrm{H}), 6.94(\mathrm{~s}, 1 \mathrm{H}), 6.04(\mathrm{dd}, J=2.5,1.5 \mathrm{~Hz}, 1 \mathrm{H}), 3.99(\mathrm{~s}, 3 \mathrm{H}), 3.97(\mathrm{~s}, 3 \mathrm{H}), 3.96(\mathrm{~s}, 3 \mathrm{H}), 3.88(\mathrm{~s}$, $3 \mathrm{H}), 3.83(\mathrm{~s}, 3 \mathrm{H}), 3.81(\mathrm{~s}, 3 \mathrm{H}), 3.61(\mathrm{br} \mathrm{s}, 2 \mathrm{H}), 3.30(\mathrm{~s}, 6 \mathrm{H}), 3.14(\mathrm{t}, J=3.5 \mathrm{~Hz}, 2 \mathrm{H}), 2.35(\mathrm{t}, J=2$ $\mathrm{Hz}, 2 \mathrm{H}$ ), 2.10 (br s, 2H), 1.25 (t, $J=7 \mathrm{~Hz}, 2 \mathrm{H}$ ); TOF-MS (electrospray) $\mathrm{m} / \mathrm{z}$ (relative intensity) 894 $\left(\mathrm{M}+\mathrm{H}^{+}, 35\right)$; Accurate mass for $\mathrm{C}_{41} \mathrm{H}_{52} \mathrm{~N}_{17} \mathrm{O}_{7}$ : calcd. 894.4236, obsd. 894.4236.

Hairpin PIP- $\gamma-I I I 3$.

Hairpin 3 was isolated as a yellow solid $(65.2 \mathrm{mg}, 30 \%), \mathrm{mp} .145-146{ }^{\circ} \mathrm{C}$ : IR (neat) 3373,2926 , 2864, 1654, 1534, 1467, 1249, $1119,752 \mathrm{~cm}^{-1} ;{ }^{1} \mathrm{H}-\mathrm{NMR}$ (DMSO-d $6,500 \mathrm{MHz}$ ) 10.38 (s, 1H), 10.19 $(\mathrm{s}, 1 \mathrm{H}), 9.84(\mathrm{~s}, 1 \mathrm{H}), 9.72(\mathrm{~s}, 1 \mathrm{H}), 9.59(\mathrm{~s}, 1 \mathrm{H}), 8.30(\mathrm{~s}, 1 \mathrm{H}), 8.03(\mathrm{~s}, 1 \mathrm{H}), 7.63(\mathrm{~s}, 1 \mathrm{H}), 7.52(\mathrm{~s}, 1 \mathrm{H})$, $7.20(\mathrm{~d}, J=1.5 \mathrm{~Hz}, 1 \mathrm{H}), 7.09$ (d, $J=1.5 \mathrm{~Hz}, 1 \mathrm{H}), 7.08$ (d, $J=2 \mathrm{~Hz}, 1 \mathrm{H}), 6.97(\mathrm{~d}, J=2 \mathrm{~Hz}, 1 \mathrm{H})$, $6.94(\mathrm{~d}, J=2 \mathrm{~Hz}, 1 \mathrm{H}), 6.04(\mathrm{dd}, J=3,4 \mathrm{~Hz}, 1 \mathrm{H}), 4.00(\mathrm{~s}, 3 \mathrm{H}), 3.96(\mathrm{~s}, 6 \mathrm{H}), 3.95(\mathrm{~s}, 3 \mathrm{H}), 3.88(\mathrm{~s}$, $3 \mathrm{H}), 3.80(\mathrm{~s}, 3 \mathrm{H}), 3.13$ (br s, $2 \mathrm{H}), 2.63(\mathrm{br} \mathrm{t}, 2 \mathrm{H}), 3.30(\mathrm{~s}, 6 \mathrm{H}), 2.35(\mathrm{t}, J=2 \mathrm{~Hz}, 2 \mathrm{H}), 2.16$ (br s, 2H), $1.80(\mathrm{t}, J=2 \mathrm{~Hz}, 2 \mathrm{H})$; TOF-MS (electrospray) $\mathrm{m} / \mathrm{z}$ (relative intensity) $895\left(\mathrm{M}+\mathrm{H}^{+}, 100\right)$; Accurate mass for $\mathrm{C}_{40} \mathrm{H}_{51} \mathrm{~N}_{18} \mathrm{O}_{7}$ : calcd. 895.4188, obsd. 895.4183.

\section{Hairpin PIP- - -IPI. 4}

Hairpin 4 was isolated as a beige solid $(62.3 \mathrm{mg}, 22 \%), \mathrm{mp} .152-157^{\circ} \mathrm{C}$ : IR (neat) $3307,2925,1653$, 1530, 1467, 1246, 1123, $750 \mathrm{~cm}^{-1}$; ${ }^{1} \mathrm{H}-\mathrm{NMR}$ (DMSO-d, $\left.500 \mathrm{MHz}\right) 10.39$ (s, 1H), 10.20 (s, 1H), $9.85(\mathrm{~s}, 1 \mathrm{H}), 9.85(\mathrm{~s}, 1 \mathrm{H}), 9.45(\mathrm{~s}, 1 \mathrm{H}), 8.33(\mathrm{~s}, 1 \mathrm{H}), 8.05(\mathrm{t}, J=5.5 \mathrm{~Hz}, 1 \mathrm{H}), 7.60(\mathrm{~s}, 1 \mathrm{H}), 7.51(\mathrm{~s}$, $1 \mathrm{H}), 7.22(\mathrm{~d}, J=1.5 \mathrm{~Hz}, 1 \mathrm{H}), 7.21(\mathrm{~d}, J=2 \mathrm{~Hz}, 1 \mathrm{H}), 7.19(\mathrm{~s}, 1 \mathrm{H}), 7.09(\mathrm{dd}, J=4,1.5 \mathrm{~Hz}, 1 \mathrm{H}), 6.97$ $(\mathrm{t}, J=2 \mathrm{~Hz}, 1 \mathrm{H}), 6.95$ (d, $J=2 \mathrm{~Hz}, 1 \mathrm{H}), 6.93(\mathrm{~d}, J=2 \mathrm{~Hz}, 1 \mathrm{H}), 6.04$ (dd, $J=4,2.5 \mathrm{~Hz}, 1 \mathrm{H}), 3.99$ (s, $3 \mathrm{H}), 3.96(\mathrm{~s}, 3 \mathrm{H}), 3.95(\mathrm{~s}, 3 \mathrm{H}), 3.88(\mathrm{~s}, 3 \mathrm{H}), 3.83(\mathrm{~s}, 3 \mathrm{H}), 3.81(\mathrm{~s}, 3 \mathrm{H}), 3.30(\mathrm{~s}, 6 \mathrm{H}), 3.22(\mathrm{br} \mathrm{t}, 2 \mathrm{H})$, 3.25 (br t, 2H), 2.29 (t, $J=3 \mathrm{~Hz}, 2 \mathrm{H}$ ), 2.18 (br s, 2H), 1.79 (br t, 2H); TOF-MS (electrospray) m/z (relative intensity) $894\left(\mathrm{M}+\mathrm{H}^{+}, 30\right)$; Accurate mass for $\mathrm{C}_{41} \mathrm{H}_{52} \mathrm{~N}_{17} \mathrm{O}_{7}$ : calcd. 894.4236, obsd. 894.4216. 
Acknowledgements: Support from the National Science Foundation, Taiho Pharmaceutical Co. (Japan), Arnold and Mabel Beckman Foundation, and GlaxoSmithKline is gratefully acknowledged. D. Harris was a Beckman Scholar.

\section{References and notes}

1. (a) P.B. Dervan, Bioorg. Med. Chem. 9, 2215 (2001) (b) P.B. Dervan and B.S. Edelson, Curr. Opin. Struct. Biol. 13, 284 (2003) (c) J.M. Gottesfeld, J.M. Turner and P.B. Dervan, Gene Expression 9, 77 (2000) (d) C. Melander, R. Bernett and J.M. Gottesfeld, J. Biotechnol. 112, 195 (2004)

2. (a) J.G. Pelton and D.E. Wemmer, J. Am. Chem. Soc. 112, 1393 (1990) (b) J.G. Pelton and D.E Wemmer, Proc. Natl. Acad.Sci. U.S.A. 86, 5723 (1989)

3. T.J. Dwyer, B.H. Geierstanger, Y. Bathini, J.W. Lown and D.E. Wemmer, J. Amer. Chem. Soc. 114, 5911 (1992).

4. (a) B.S. Reddy, S.K. Sharma and J.W. Lown, Curr. Med. Chem. 8, 475 (2001) (b) M.L. Kopka, D.S. Goodsell, G.W. Han, T.K. Chiu, J.W. Lown and R.E. Dickerson, Structure 5, 1033 (1997).

5. X.L. Yang, R.B. IV Hubbard, M. Lee, Z.H. Tao, H. Sugiyama and A.H.J. Wang, Nucl. Acids Res. 27, 4183 (1999).

6. X.L. Yang, C. Kaenzig, M. Lee and A.H.J. Wang, Eur. J. Biochem. 263, 646 (1999).

7. E.R. Lacy, N.M. Le, C.A. Price, M. Lee and D.W. Wilson, J. Amer. Chem. Soc. 124, 2153 (2002)

8. (a) M.E. Parks, E.E. Baird and P.B. Dervan, J. Am. Chem. Soc. 118, 6153 (1996) (b) M.E. Parks, E.E. Baird and P.B. Dervan, J. Am. Chem. Soc. 118, 6147 (1996) (c) S.E. Swalley, E. E. Baird and P.B. Dervan, J. Am. Chem. Soc. 121, 1113 (1999) (d) S.E. Swalley, E.E. Baird, and P.B. Dervan, J. Am. Chem. Soc. 119, 6953 (1997)

9. (a) H. Matsuda, N. Fukuda, T. Ueno, Y. Tahira, H. Ayame, W. Zhang, T. Bando, H. Sugiyama, S. Saito, K. Matsumoto, H. Mugishima and K. Serie, J. Am. Soc. Nephrol. 17, 422 (2006) (b) Y.M. Lai, N. Fukuda, T. Ueno, H. Matsuda, S. Saito, K. Matsumoto, H. Ayame, T. Bando, H. Sugiyama, H. Mugishima and K. Serie, J. Pharmacol. Exp. Ther. 315, 571 (2005)

10. J.H. Henry, N.M. Le, B. Nguyen, C.M Howard, A.L. Bailey, K.L. Buchmueller, M. Kotecha, D. Hochhauser, J. A. Hartley, D.W. Wilson and M. Lee, Biochemistry 43, 12249 (2004)

11. E.E. Baird and P.B. Dervan, J. Am. Chem. Soc. 118, 6141 (1996)

12. T.; Matsumoto, K. Toyoka, E. Nishiwaki and M. Shibuya, Heterocycles 31, 1629 (1990)

13. Acid chloride 9 was prepared as follows: 4-nitro-l-methylimidazole carboxylic acid ${ }^{14}(10,1$ equiv.) was dissolved in dry THF. Oxalyl chloride $(1: 1$ ratio, $1.5 \mathrm{~mL}$ of each per $200 \mathrm{mg}$ of acid) was added, and the solution refluxed for $50 \mathrm{~min}$. Acid chloride 19 was prepared in a similar manner: 4-nitro-1-methylpyrrole carboxylic acid ${ }^{16}(\mathbf{2 0}, 1$ equiv.) was dissolved in $\mathrm{SOCl}_{2}(3 \mathrm{~mL}$ per $200 \mathrm{mg}$ of acid) and the solution refluxed for $10 \mathrm{~min}$. In both cases, the excess solvent was removed via aspiration, and the residue co-evaporated twice with dry $\mathrm{CH}_{2} \mathrm{Cl}_{2}(3 \times 2 \mathrm{~mL})$ and the isolated compound used directly.

14. J.W. Lown and K. Krowicki, J. Org. Chem. 52, 3493 (1987)

15. (a) M. Lee, A.L. Rhodes, M.D. Wyatt, S. Forrow and J.A. Hartley, Biochemistry 32, 4237 (1993) (b) M. Lee, A.L. Rhodes, M.D. Wyatt, S. Forrow, M. D'Incalci and J.A. Hartley, J. Med. Chem. 36, 863 (1993) (c) E.R. Lacy, N.M. Le, C.A. Price, M. Lee and W.D. Wilson, J. Am. Chem. Soc. 124, 2153 (2002)

16. J.W. Lown and K. Krowicki, J. Org. Chem. 50, 3774 (1985).

Received on December 5, 2006. 
